This item was submitted to Loughborough's Research Repository by the author.

Items in Figshare are protected by copyright, with all rights reserved, unless otherwise indicated.

\title{
Spinal cord injury level influences acute plasma caffeine responses
}

PLEASE CITE THE PUBLISHED VERSION

http://dx.doi.org/10.1249/MSS.0000000000001108

\section{PUBLISHER}

(c) the American College of Sports Medicine. Published by Lippincott, Williams \& Wilkins

\section{VERSION}

AM (Accepted Manuscript)

\section{PUBLISHER STATEMENT}

This work is made available according to the conditions of the Creative Commons Attribution-NonCommercialNoDerivatives 4.0 International (CC BY-NC-ND 4.0) licence. Full details of this licence are available at: https://creativecommons.org/licenses/by-nc-nd/4.0/

\section{LICENCE}

CC BY-NC-ND 4.0

\section{REPOSITORY RECORD}

Graham-Paulson, Terri S., Thomas A.W. Paulson, Claudio Perret, Keith Tolfrey, Philip Cordery, and Victoria L. Goosey-Tolfrey. 2019. "Spinal Cord Injury Level Influences Acute Plasma Caffeine Responses". figshare. https://hdl.handle.net/2134/22781. 
1 Spinal cord injury level influences acute plasma caffeine responses.

2 Terri Susan Graham-Paulson ${ }^{1}$, Thomas Andrew William Paulson ${ }^{1}$, Claudio Perret ${ }^{2}$, $3 \quad$ Keith Tolfrey $^{3}$, Philip Cordery ${ }^{3}$ and Victoria Louise Goosey-Tolfrey ${ }^{1, *}$.

$4{ }^{1}$ Peter Harrison Centre for Disability Sport, School of Sport, Exercise and Health Sciences, 5 Loughborough University, Leicestershire, UNITED KINGDOM.

${ }^{2}$ Swiss Paraplegic Centre, Institute of Sport Medicine, Nottwil, SWITZERLAND.

${ }^{3}$ School of Sport, Exercise and Health Sciences, Loughborough University, Leicestershire, UNITED KINGDOM.

9

*Correspondence:

Email: v.l.tolfrey@lboro.ac.uk

Tel.: +44 (0)1509 226386

Address: Peter Harrison Centre for Disability Sport, Loughborough University, School of Sport, Exercise and Health Sciences, Epinal Way, Loughborough, LE113TU, UK

\section{Abstract}

Purpose. To investigate the absorption curve and acute effects of caffeine at rest in individuals with no spinal cord injury (SCI), paraplegia (PARA) and tetraplegia (TETRA). Methods. Twenty-four healthy males (8 able-bodied (AB), 8 PARA and 8 TETRA) consumed $3 \mathrm{mg} \cdot \mathrm{kg}^{-1}$ caffeine anhydrous (CAF) in a fasted state. Plasma caffeine [CAF], glucose, lactate, free-fatty acid [FFA] and catecholamine concentrations were measured during a 150 min rest period. Results. Peak [CAF] was greater in TETRA (21.5 $\mu \mathrm{M})$ compared to AB $(12.2 \mu \mathrm{M})$ and PARA $(15.1 \mu \mathrm{M})$, and mean peak [CAF] occurred at 70, 80 and 80 min, respectively. Moderate and large ES were revealed for TETRA compared to 
curve. Large inter-individual responses were apparent in SCI groups. The change in plasma catecholamine concentrations following CAF did not reach significance $(\mathrm{p}>0.05)$ however

27 both adrenaline and noradrenaline concentrations were lowest in TETRA. Significant 28 increases in $[F F A]$ were seen over time $(\mathrm{p}<0.0005)$ but there was no significant influence of 29 SCI level. Blood lactate concentration reduced over time $(\mathrm{p}=0.022)$ whereas blood glucose 30 concentration decreased modestly $(\mathrm{p}=0.695)$, and no difference between groups was seen 31 ( $>>0.05)$. Conclusion. Level of SCI influenced the caffeine absorption curve and there was 32 large inter-individual variation within and between groups. Individual curves should be 33 considered when using caffeine as an ergogenic aid in athletes with an SCI. The results 34 indicate TETRA should trial low doses in training and PARA may consider consuming 35 caffeine greater than 60 min prior to exercise performance. The study also supports caffeine’s 36 direct effect on adipose tissue, which is not secondary to catecholamine release.

37 Keywords: adrenaline; noradrenaline; free fatty acid; ergogenic; wheelchair athletes 
Supplementation with caffeine (3-6 $\left.\mathrm{mg} \cdot \mathrm{kg}^{-1}\right)$ can improve long and short-term endurance performance $(7,9)$ in able-bodied $(A B)$ participants. However, there is a paucity of research on the effects of caffeine on exercise performance in physically impaired populations e.g. persons with a spinal cord injury (SCI). While current evidence is equivocal, a beneficial effect of caffeine (4-6 mg $\cdot \mathrm{kg}^{-1}$ body mass (BM) in capsule form) on short-term wheelchair propulsion exercise has been reported $(5,13)$. These studies highlighted that there was great inter-individual variability in wheelchair performance responses during a $1500 \mathrm{~m}$ time trial, 4 min maximal push and repeated sprints, especially in individuals with an SCI. The authors highlighted the potential for slower caffeine absorption due to delayed gastrointestinal (GI) transit times and prolonged gastric emptying (GE), especially in those with a cervical lesion level (18). Understanding an individual's time to peak caffeine concentration has been shown to have little impact on prolonged $\mathrm{AB}$ endurance cycling performance (34) but is likely to be important prior to short-term upper-body exercise (UBE), and may require further consideration in persons with an SCI.

Both metabolic and physiological functions are altered in individuals with an SCI, and the level and completeness of injury has been shown to influence drug pharmacokinetics $(15,23)$. A review of the literature by Mestre et al. (23) indicated that the delayed absorption seen in some individuals with an SCI increased the time to achieve the required therapeutic dose. One drug reportedly affected by delayed GE and decreased GI motility is theophylline (32), which can be used by individuals with an SCI to help treat bradycardia or to promote the recovery of hemidiaphragmatic function. Diminished bioavailability could result in underestimating the load and maintenance dose of theophylline in individuals with tetraplegia (32). As a methylxanthine drug, theophylline has similar pharmacodynamic actions to caffeine (28) and it has also been linked to improved endurance performance $(14,22)$. There 
is therefore reason to believe that caffeine absorption may also be delayed in persons with an SCI. In disagreement however, Van Soeren et al. (37) suggested that the time to peak caffeine concentration $\left(6 \mathrm{mg} \cdot \mathrm{kg}^{-1}\right)$ in individuals with tetraplegia $(\sim 47 \mu \mathrm{M}$ at $40 \mathrm{~min}(\mathrm{n}=6))$ did not differ to those of AB individuals. The authors however could not assess the influence of SCI lesion level on caffeine absorption because there was no direct control group and only two individuals with paraplegia. They also did not report individual participant data, which may help to explain inter-individual performance responses. Flueck et al. (4) measured plasma caffeine concentrations (median) at $60 \mathrm{~min}$ only in $\mathrm{AB}$ individuals $\left(45.1 \mu \mathrm{mol} \cdot \mathrm{L}^{-1}\right.$ ) and individuals with paraplegia $\left(\sim 54 \mu \mathrm{mol} \cdot \mathrm{L}^{-1}\right)$ and tetraplegia $\left(66.1 \mu \mathrm{mol} \cdot \mathrm{L}^{-1}\right)$. It therefore remains difficult to determine whether the time course of caffeine absorption differs based on an individual's SCI level.

Numerous mechanisms of action have been proposed to help explain the beneficial effects of an acute dose of caffeine on exercise performance. Current research suggests the main mechanism at physiological caffeine doses is the blockade of central nervous system (CNS) adenosine receptors, which indirectly effects-affects neurotransmitter release (19) to increase arousal, alertness and attention. Individuals with tetraplegia are therefore an interesting study population given the reduced sympathetic activity caudal to the lesion level and associated impaired catecholamine response (27). The study of this population has lent support to the hypothesis that caffeine can have a direct effect on tissues following reports of adrenalineindependent free fatty acid (FFA) mobilisation (37). No study has directly investigated the acute effects of caffeine in a group of individuals with no SCI, paraplegia and tetraplegia. Hence, the current study aimed to explore the time course of caffeine absorption and its effects at rest in these three groups, with the aim of providing safe and accurate recommendations for its use as an ergogenic aid by individuals with an SCI. It wais 
hypothesised that caffeine absorption will be delayed in individuals with tetraplegia compared to those with paraplegia and no SCI.

\section{Methods}

Participants. Twenty-four healthy recreationally active males (8 able-bodied controls (AB), 8 individuals with paraplegia (PARA) and 8 with tetraplegia (TETRA)) provided informed consent to participate in the current study. Participants were classified using the American Spinal Injury Association (ASIA) scale (20). A health screening questionnaire was completed by all participants and individuals were excluded if any of their medication had known interactions with caffeine. Average daily caffeine intake was assessed using a modified version of the caffeine consumption questionnaire (21). All procedures were approved by the University Ethical Advisory Committee and performed following the Declaration of Helsinki. Participants’ characteristics are shown in Table 1.

Procedures. In the days prior to visiting the laboratory, participants maintained their normal dietary and activity patterns_(light-moderate intensity exercise only) and their individual medication regimes. Participants were provided with a list of caffeine containing foods and drinks, and were asked to abstain from consumption in the $36 \mathrm{~h}$ preceding their laboratory visit. Participants were also asked to refrain from alcohol consumption for $24 \mathrm{~h}$ prior to their $\underline{\text { visit. }}$

Participants arrived at the laboratory (between 08:00 and -10:-00 - after fasting from 21:00 the previous eveningin afollowing an overnight fast fasted state(no food intake after 21:00). Water consumption was encouraged to help ensure the participant arrived euhydrated. On arrival participants were asked to void their bladder, if necessary, prior to lying in a semisupine position on a laboratory bed. Participants were asked to report any side-effects to the investigators immediately at any point during the trial. A cannula (Venflon, Becton 
Dickinson, Helsinborg, Sweden) was inserted into an antecubital vein for subsequent venous sampling. The cannula was kept patent using 5-10 ml sodium chloride (0.9\%) after each blood sample.

After a minimum of 15 min rest, a baseline venous blood sample was taken. Participants then consumed cellulose capsules (Bulk Powders, Colchester, UK) containing $3 \mathrm{mg} \cdot \mathrm{kg}^{-1} \mathrm{BM}$ caffeine anhydrous (My Protein, Northwich, UK), which were filled manually by the investigators to the nearest $0.1 \mathrm{mg}$. Participants remained rested for $150 \mathrm{~min}$ during which a further 9 blood samples were taken. The blood sampling schedule can be seen in Figure 1. After the final blood sample, participants were again asked once more-to record-whether they experienced any side-effects during the experimental trial.

Blood sampling and analysis. Blood samples were immediately separated into tubes that contained the relevant preservative(s). At every sampling time-point $5 \mathrm{ml}$ blood was added to an EDTA K2 vacutainer for subsequent plasma caffeine concentration ([CAF]) analysis. A 20 $\mu \mathrm{l}$ blood sample was removed and analysed in duplicate for blood lactate ([BLa]) and glucose ([GLU]) concentrations using an automatic analyser (Biosen C-Line, EKF Diagnostic GmbH, Barleben, Germany). For catecholamine and FFA analysis (baseline, 60, 90 and $150 \mathrm{~min}$ ), a further $10 \mathrm{ml}$ of blood was dispensed into two lithium-heparin tubes containing $37.5 \mu \mathrm{l}$ of EGTA-Glutathione for the subsequent analysis of plasma adrenaline ([A]), noradrenaline ([NA]) and FFA ([FFA]) concentrations. In addition, $25 \mu \mathrm{l} \mathrm{of} 3 \mathrm{mg} \cdot \mathrm{ml}^{-1}$ tetrahydrolipstatin (THL) was added to the tube for [FFA] analysis. All tubes were centrifuged at $1000 \mathrm{~g}$ for 10 min at $4^{\circ} \mathrm{C}$ as previously described (12). Plasma samples were aliquoted into Eppendorfs and stored at $-80^{\circ} \mathrm{C}$ until analysis.

Plasma [CAF] was analysed using high-performance liquid chromatography (HPLC) as described by Holland et al. (16) with the following minor modifications; prior to injection 
onto the HPLC column each sample was individually filtered (Mini-UniPrep syringeless filters, Fisher Scientific, UK) and no guard column was used. The method produced a coefficient of variation (CV) of 1.06\% (range $0.24-1.45 \%$ ).

Plasma [A] and [NA] were also determined using HPLC as previously described by Forster \& Macdonald (6). A plasma volume of $500 \mu \mathrm{l}$ was used for analysis. The method produced CVs of 0.31 and $0.17 \%$ for $[\mathrm{A}]$ and $[\mathrm{NA}]$ respectively.

Plasma was analysed enzymatically for [FFA] using an in vitro enzymatic colorimetric method (Wako Instrument kit) and a Pentra 400 analyser (Horiba Medical, California, USA). The method produced an intra-assay CV of 1.68 and $1.28 \%$ for high and low FFA quality controls (QC) (4 repeats of the QC samples at intervals during the analysis).

\section{Statistical analyses}

Data were analysed using the IBM Statistics Software Package for the Social Sciences (SPSS) version 22 (IBM Corporation, New York, USA). The trapezium rule was used to calculate the total area under the variable versus time curve for [CAF] (TAUC-CAF), [FFA] (TAUC-FFA), [A] (TAUC-A) and [NA] (TAUC-NA). The incremental area under the plasma concentration versus time curve for [FFA] (iAUC-FFA), [A] (iAUC-A) and [NA] (iAUC-NA) was also calculated using the same method after adjusting for baseline concentrations.

Normal distribution was checked using Shapiro-Wilk tests and the data are presented as mean \pm SD. Data for [FFA] were not normally distributed and were log transformed prior to analysis. These data are presented as geometric mean (95\% confidence intervals (CI)) and analysis is based on the ratios of geometric means and 95\% CI for ratios. Homegeneity of variances was confirmed by Mauchly's test of sphericity, and where the sphericity assumption was violated, the Greenhouse Geisser correction was applied to the degrees of freedom. 
Repeated measures analysis of variance (ANOVA) for group and time were used to examine differences between [FFA], [A], [NA], [Bla] and [GLU].

An analysis of covariance (ANCOVA) was used to examine differences between [CAF], with daily caffeine consumption (low $<50 \mathrm{mg} \cdot \mathrm{d}^{-1}$, moderate $50-250 \mathrm{mg} \cdot \mathrm{d}^{-1}$ and high $>250 \mathrm{mg} \cdot \mathrm{d}^{-1}$ ) as a covariate. One-way repeated measures ANOVAs were used to analyse TAUC and iAUC data. Planned simple and difference contrasts were applied to explain any significant results. Statistical significance was accepted at $p \leqslant 0.05$ and absolute standardised effect sizes (ES) are included to supplement important findings. An ES of 0.2 was considered small, 0.5 moderate and 0.8 large according to Cohen (3). Due to incomplete data sets (e.g. insufficient blood flow or a cannula change) the number of participants included in each analysis differs. Data sets were A (7/6/7), NA (7/7/8), FFA (7/7/7), Bla (5/5/6) and GLU (8/6/8) for AB, PARA and TETRA groups, respectively.

Power analysis was performed using the [CAF] observed in 3 groups of participants with no SCI, paraplegia and tetraplegia 60 min post-ingestion of $6 \mathrm{mg} \cdot \mathrm{kg}^{-1}$ caffeine (46.4 (6.8), 55.3 (19.8) and 64.1 (6.9) $\mu \mathrm{M}$, respectively) (4). The a priori analysis, conducted in G*Power 3.1, revealed that six participants would be required in each group to detect a similar change in [CAF] with ES of $0.59,0.66$ and 2.74 (4), 90\% power, and an $\alpha$ of $5 \%$. Given the novel nature of this investigation and the heterogeneity of the population, an additional two participants per group were recruited to increase statistical power $(n=8)$.

\section{Results}

\section{Plasma caffeine}

At baseline, [CAF] was either undetectable or very low, which indicates that all participants adhered to the withdrawal guidelines. Differences over time and across groups were revealed (main effect time $\mathrm{p}<0.0005$; main effect group $\mathrm{p}=0.026$; time by SCI level interaction $\mathrm{p}=$ 
0.019) (Figure 2). Planned simple contrasts revealed these group differences occurred between AB and TETRA ( $p=0.017$ ), whereas no difference was observed between AB and PARA ( $p=0.913)$. Peak [CAF] in TETRA was significantly greater than AB $(p=0.008)$ yet non-significantly $(\mathrm{p}=0.058)$, but meaningfully $(\mathrm{ES}=0.9)$ greater than PARA $(21.5 \pm 7.0$, $12.2 \pm 2.3$ and $15.1 \pm 8.1 \mu \mathrm{M}$, respectively). Time to peak [CAF] varied greatly between individuals but group means were $80 \mathrm{~min}$ for $\mathrm{AB}$ and PARA, and $70 \mathrm{~min}$ for TETRA. There was no influence of habitual caffeine use on $[\mathrm{CAF}](\mathrm{p}=0.943)$.

No significant difference in TAUC-CAF was observed between groups $(\mathrm{p}=0.135$; AB $3.74 \pm$ $0.96 \mu \mathrm{M}$, PARA $4.62 \pm 3.12 \mu \mathrm{M}$, and TETRA $6.08 \pm 2.15 \mu \mathrm{M}$ ). However, small (AB vs. PARA, ES $=0.38)$, moderate (PARA vs. TETRA, ES = 0.55) and large (AB vs. TETRA, ES = 1.14) ES were apparent.

Seven participants (3 AB/2 PARA/2 TETRA) reported adverse effects prior to/during the first 30 min of testing (headache/light-headed (2)) and during testing- (struggling with quick decision making (1), tingling arm (1), twitching eye (1)) and five participants reported feeling more alert.

\section{Plasma catecholamines (adrenaline and noradrenaline)}

199

200

201

202

203

204

205

206

All catecholamine analysis excluded the participant with a T4 lesion level due to a missed sample and hence statistical analysis for PARA was calculated based on injuries at or below T6/7. The change in [A] over the course of the resting protocol did not reach statistical significance, $_{2}$ but did differ between groups (main effect of time $\mathrm{p}=0.088$; main effect of group $\mathrm{p}=0.027$; time by SCI level $p=0.618$ ) (Figure 3 ). Planned difference contrasts revealed these group differences occurred between PARA and TETRA ( $p=0.019)$ only. There was no significant difference in TAUC-A $(p=0.075)$ between groups $(A B \quad 0.43 \pm 0.17$ $\mathrm{nmol} \cdot \mathrm{L}^{-1}$, PARA $0.57 \pm 0.22 \mathrm{nmol} \cdot \mathrm{L}^{-1}$, and TETRA $0.22 \pm 0.10 \mathrm{nmol} \cdot \mathrm{L}^{-1}$ ) though ES were 
large for both $\mathrm{AB}(\mathrm{ES}=2.02)$ and PARA $(\mathrm{ES}=1.04)$ compared to TETRA. There was no difference in iAUC-A $(p=0.733)$.

The [NA] did not change significantly during the $150 \mathrm{~min}$ protocol $(\mathrm{p}=0.423)$ but did differ between groups $(\mathrm{p}=0.003)$, and no interaction was evident $(p=0.772)$. Planned difference contrasts revealed these group differences occurred between AB and TETRA ( $p=0.001)$, and PARA and TETRA $(\mathrm{p}=0.006)$, but no significant difference was observed between AB and PARA $(p=0.505)$. There was a significant difference in TAUC-NA $(p=0.003)$ between groups $\left(\mathrm{AB} 4.04 \pm 0.92 \mathrm{nmol} \cdot \mathrm{L}^{-1}\right.$, PARA $3.68 \pm 1.01 \mathrm{nmol} \cdot \mathrm{L}^{-1}$, and TETRA $2.01 \pm 1.21$ $\left.\mathrm{nmol} \cdot \mathrm{L}^{-1}\right)$. Small $(\mathrm{AB}$ vs. PARA, ES $=0.38)$ and large $(\mathrm{AB}$ vs. TETRA, $\mathrm{ES}=1.89$, and PARA vs. TETRA, ES = 1.50) ES were revealed. However, no significant difference in iAUC-NA was observed $(\mathrm{p}=0.827)$.

\section{Plasma FFA, lactate and glucose}

Differences in [FFA] were observed over time and between groups; with the latter not reaching significance, but displaying a large effect-however the latter failed to reach significance $(\mathrm{ES}=0.80)$ (main effect time $\mathrm{p}<0.0005$; main effect group $\mathrm{p}=0.054$; time by group interaction $\mathrm{p}=0.035)$. Means (95\% CI) $[\mathrm{FF} A]$ wereas $51 \%(-42$ to $-24 \%), 64 \%(-47$ to $-30 \%$ ) and $84 \%$ (-54 to -37\%) higher than baseline at 60,90 and 150 min, respectively.

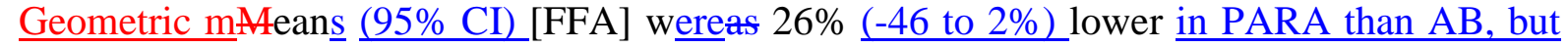
and-9\% higher than AB in PARA (95\% CI - 2-46 to 862\%) in and-TETRA than AB (95\% CI34-21 to 27 $\underline{50} \%$ ), respectively; - furthermore, Mean-[FFA] results-wasere 47\% (6 to 103\%) higher in TETRA thaneompared to PARA (95\% CI - 51으 to -6103\%)-(Figure 3). The interaction indicated that while PARA experienced only a marginal increase in [FFA] from baseline to $150 \mathrm{~min}\left(\Delta \sim 0.13 \mathrm{mmol} \cdot \mathrm{l}^{-1}\right), \mathrm{AB}$ and TETRA increased to a greater extent over the course of the protocol ( $\Delta \sim 0.36$ and $0.31 \mathrm{mmol} \cdot \mathrm{l}^{-1}$, respectively). 
Although the main effect for No significant-differences in TAUC-FFA was not significantebserved $(p=0.072)$, the effect sizes ranged from small yet moderate (AB vs.

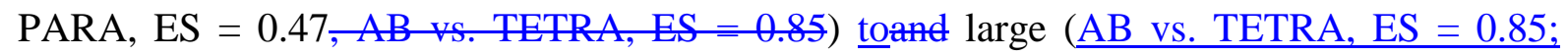
PARA vs. TETRA, ES = 1.16) ES were revealed. No significant difference in iAUC-FFA was observed $(p=0.357)$.

Differences in [Bla] were observed over time but not between groups (main effect time $\mathrm{p}=$ 0.022; main effect group $\mathrm{p}=0.463$; time by group interaction $\mathrm{p}=0.065$ ). Planned difference contrasts revealed a significant decrease in [Bla] between baseline and 60 min $(p-0.049)$, and between 90 and $150 \mathrm{~min}(\mathrm{p}<0.0005)$. No significant difference in [GLU] was seen over the course of the $150 \mathrm{~min}$ protocol $(\mathrm{p}=0.695)$ or between groups $(\mathrm{p}=0.983)$.

\section{Discussion}

The current study is the first to report large inter-individual differences in caffeine absorption within and between groups when separated for level of SCI (AB, PARA and TETRA). Consequently, dosage and timing recommendations provided to individuals with an SCI may need to be adapted from the $\mathrm{AB}$ literature. In addition, the pattern of caffeine absorption differs in TETRA compared to AB and PARA. There were small differences in [A], [NA] and [FFA] between the AB and SCI groups, which were non-significant when baseline values were accounted for using the incremental area under the curve. No differences in [Bla] and [GLU] were seen between groups.

\section{Plasma caffeine}

Participant's [CAF] increased in all three groups following the ingestion of $3 \mathrm{mg} \cdot \mathrm{kg}^{-1}$ caffeine. The [CAF] in $\mathrm{AB}$ at $60 \mathrm{~min}(10.8 \pm 3.1 \mu \mathrm{M})$ is in line with that reported 60 min postingestion of 2, 3 and $4 \mathrm{mg} \cdot \mathrm{kg}^{-1}$ caffeine (5.7, 15 and $14.6 \mu \mathrm{M}$, respectively) $(11,33)$. This 
study is the first to investigate the caffeine absorption curve in a group of participants with paraplegia. In agreement with the hypothesis, the PARA results do not differ from the AB responses at $60 \mathrm{~min}(11.1 \pm 7.9 \mu \mathrm{M})$ and both groups reached mean peak [CAF] at $80 \mathrm{~min}$. The TETRA responses were significantly greater than $A B$ and the mean peak [CAF] was reached 10 min earlier (70 min). Flueck et al. (4) also reported a higher [CAF] 60 min postingestion of $\sim 6 \mathrm{mg} \cdot \mathrm{kg}^{-1}$ caffeine in individuals with tetraplegia compared to those with paraplegia (66.1 and $45.1 \mu \mathrm{M}$, respectively). Interestingly, Van Soeren et al. (37) also reported a high peak [CAF] of $46.7 \pm 5.0 \mu \mathrm{M}$ in individuals with tetraplegia ( $\mathrm{n}=6)$ yet this was reached after only $40 \mathrm{~min}$ post-ingestion of $6 \mathrm{mg} \cdot \mathrm{kg}^{-1}$ caffeine. The current study therefore adds further support to reports of higher [CAF] in TETRA compared to individuals with lower lesion levels and no spinal injury. Furthermore, the data also highlight the large variability that exists within each group. Based on the current study there does not appear to be an influence of habitual caffeine use on the participants' [CAF] in response to a single dose, as seen previously (1). Seven participants reported adverse effects which were likely a result of withdrawal (headache), fasting (light headed) and CAF (tingling arm, twitching eye and struggling to make quick decisions). All symptoms were mild and only lasted for a short duration. The $3 \mathrm{mg} \cdot \mathrm{kg}^{-1}$ caffeine dose is therefore deemed safe in this population.

An interaction effect occurred due to the sharp increase in [CAF] in TETRA while both AB and PARA groups [CAF] increased gradually followed by a plateau. The rapid increase in [CAF] in TETRA means that the hypothesis of slowed absorption in this population can be rejected. The sharp rise may be due to a number of factors. Firstly, individuals with tetraplegia have a smaller blood volume compared to AB individuals (17) due to atrophy of the musculature and vessels of the lower limbs. This reduced blood volume may result in a falsely large [CAF] in TETRA following the administration of a standardised dose per kilogram body mass. Secondly, following a cervical or thoracic SCI sympathetic outflow to 
the liver is also disrupted, which in turn can lead to hepatic pathology (30). The liver is innervated by both sympathetic and parasympathetic nerves, and the sympathetic splanchnic nerves originate from neurons which are located in the spinal column (T7-T12) (38). Acute changes to the liver occur due to the complete (cervical level) and partial (thoracic level) disruption to the descending control of sympathetic neurons innervating the organ (30). It has been proposed that abnormal liver function may affect the metabolism and bioavailability of drugs $(23,30)$. The half-life of many drugs can be prolonged in individuals with an SCI who display suboptimal liver function and slow renal clearance $(23,30)$. Serum caffeine half-life has also been shown to be severely prolonged in individuals with compromised liver function e.g. those with alcoholic hepatic liver disease (36). The half-life of caffeine in healthy individuals is $\sim 4-6 \mathrm{~h}$ (1). This may help explain the sharp rise to peak [CAF] in TETRA (slowed metabolism) which remains higher than AB and PARA (slowed renal clearance). This TETRA response indicates that individuals with a cervical SCI may consider using a lower dose of caffeine to produce similar [CAF] as AB and PARA while avoiding any potential side-effects that are reported anecdotally and in previous research (13). It also suggests that individuals with a high lesion level may need to consider reducing the frequency of caffeine intake to prevent the potential negative effects of high doses of caffeine e.g. nervousness, jitters, restlessness, sleeplessness and irritability.

The TAUC-CAF did not statistically differ between groups yet a large ES of 1.14 was evident between AB and TETRA. Large inter-individual responses were evident in both SCI groups (Figure 2) due to the heterogeneous nature of this population. The equivocal findings regarding the beneficial effects of caffeine during short-term exercise performance (4) may be partly explained by these inter-individual differences, highlighted by the current PARA and TETRA responses. Examination of individual data within PARA reveals some interesting findings. Participant 9 (L1 lesion; ASIA B) produced a similar curve to the AB participants, 
with a peak (albeit larger) at 45 min followed by a steady decline. However, caffeine did not appear in the bloodstream of participant 10 (T7 lesion; ASIA B) until 70 min and continued to rise for the remaining 80 min. Hence the implementation of a standard caffeine protocol whereby caffeine is administered 60 min prior to short-term exercise performance would result in participant 10 exhibiting a [CAF] associated with a placebo dose at the commencement of exercise. For short-term exercise performance it is therefore recommended that an athlete with an SCI determines their individual absorption curve to produce individualised dose and timing recommendations. If this is impractical it is recommended that caffeine is provided to individuals with paraplegia earlier to ensure it enters the bloodstream prior to exercise performance. Research into the use of caffeine gum or mouth rinse is emerging yet the evidence of a consistent positive effect is currently limited $(26,29)$. Consuming caffeine in this format allows direct absorption into the bloodstream through the buccal mucosa and may eliminate any potential issues regarding caffeine absorption in individuals with an SCI.

Body mass and habitual caffeine intakes were similar between all three groups, but PARA were significantly older than AB (Table 1). However, previous research has suggested that age is not associated $(p>0.612)$ with $C_{\max }$ or time to $C_{\max }$ following caffeine ingestion (Skinner et al., 2014) nor does it affect gastric emptying (Kao et al., 1999). Furthermore, no significant correlations were observed between age and $\mathrm{C}_{\max }(\mathrm{r}=0.07)$ or time to $\mathrm{C}_{\max }(\mathrm{r}=$ $\underline{0.11) \text { within the current cohort. }}$

\section{Plasma catecholamines (adrenaline and noradrenaline)}

Resting plasma catecholamine concentrations did not significantly increase over the course of the 150 min protocol in any group (Figure 3). In contrast, Flueck et al. (4) and Van Soeren et al. (37) reported increases in adrenaline in both $\mathrm{AB}$ individuals and individuals with 
328 paraplegia, which may in part be due to the larger $6 \mathrm{mg} \cdot \mathrm{kg}^{-1}$ dose administered in these studies. In line with previous findings, baseline catecholamine concentrations were lower in TETRA compared to $\mathrm{AB}$ and PARA due to the impaired sympathetic activation of the nervous system $(27,31)$.

\section{Plasma FFA, lactate and glucose}

333 Mean resting [FFA] increased over time from $0.36 \pm 0.19 \mathrm{mmol} \cdot \mathrm{L}^{-1}$ at baseline to $0.61 \pm 0.25$ at 150 min, in agreement with previous research in an AB and SCI population $(10,37)$. In the absence of a catecholamine response, the current results lend further support for a direct effect of caffeine on human tissue, specifically adipocytes. The majority of research suggests that FFA availability does not result in greater FFA oxidation and therefore does not alter substrate use at rest or during exercise $(12,24)$. It is also unlikely to aid performance during short-term upper-body exercise where participants/athletes predominantly work anaerobically, and therefore utilise carbohydrate as the main substrate.

Baseline [FFA] was higher in TETRA than AB or PARA (Figure 3). The lack of muscle innervation of paralysed lower limbs in individuals with an SCI leads to rapid muscle atrophy and a reduction in resting metabolic rate (25). Alongside poor nutritional choices and a disruption in the secretion of anabolic hormones, these changes can result in an increase in fat mass (35). An expanding fat mass which releases more FFA and a potential reduction in FFA clearance leads to increased plasma [FFA] (2). The [FFA] responses only significantly differed between the two SCI groups (PARA vs. TETRA). One possible explanation for this could be the difference in the group’s time since injury (PARA $4.3 \pm 4.3 \mathrm{yr}$ and TETRA 12.2 $\pm 6.3 \mathrm{yr}$ ) which has been positively associated with loss of lean tissue and increased fat mass 
collected to enable a greater understanding of the [FFA] responses and whether substrate use

Many studies report an increase in [Bla] during exercise following the ingestion of caffeine, sometimes in the absence of increased workload/speed/power. At rest however, the current data show [Bla] decreased slightly over the course of the 150 min protocol, which is in line with previous data (37). On the other hand, [GLU] decreased modestly (non-significantly) during the current protocol, as previously reported (24) and is unlikely a result of caffeine ingestion.

\section{Conclusion}

The current study demonstrates that there is large inter-individual variability in caffeine absorption in individuals with an SCI and that this should be assessed prior to making specific recommendations for its use. Individuals with tetraplegia may consider using a lower dose and individuals with paraplegia may consider consuming supplementary caffeine earlier than the 60 min recommended to $\mathrm{AB}$ individuals.

\section{Acknowledgements}

The authors would like to thank all the participants for taking part in the study, the University of Nottingham School of Life Sciences for performing the plasma catecholamine and free fatty acid concentration analysis, and The Peter Harrison Centre for Disability Sport for their support.

\section{Conflicts of interest}

371 The authors declare no conflict of interest. The results of the present study do not constitute

372 endorsement by ACSM. The authors declare that the results of the study are presented clearly, honestly, and without fabrication, falsification, or inappropriate data manipulation. 


\section{References}

1. Bell DG, McLellan TM. Exercise endurance 1, 3, and $6 \mathrm{~h}$ after caffeine ingestion in caffeine users and nonusers. J Appl Physiol. 2002;93:1227-1234.

2. Bjorntorp P, Bergman H, Varnauskas E. Plasma free fatty acid turnover rate in obesity. Act Med Scand. 1969;185:351-356.

3. Cohen JA. A power primer. Psychol Bulletin. 1992;112(1):155-159.

4. Flueck JL, Lienert M, Schaufelberger F, Krebs J, Perret C. Ergogenic effects of caffeine consumption in a 3 min all-out arm crank test in paraplegic and tetraplegic compared to able-bodied individuals. Int J Sport Nutr Ex Metab. 2015;25(6):584-593.

5. Flueck JL, Mettler S, Perret C. Influence of caffeine and sodium citrate ingestion on 1500 m exercise performance in elite wheelchair athlete: A pilot study. Int J Sport Nutr Exerc Metab. 2014;24:296-304.

6. Forster CD, Macdonald IA. The assay of the catecholamine content of small volumes of human plasma. Biomed Chromatogr. 1999;13:209-215.

7. Ganio MS, Klau JF, Casa DJ, Armstrong LE, Maresh CM. Effect of caffeine on sportspecific endurance performance: a systematic review. J Strength Cond Res. 2009;23(1):315-324.

8. Gorgey AS, Wells KM, Austin TL. Adiposity and spinal cord injury. World J Orthop. 2015;6(8):567-576.

9. Graham TE. Caffeine and exercise: metabolism, endurance and performance. Sports Med. 2001;31(11):785-807.

10. Graham TE, Helge JW, MacLean DA, Kiens B, Richter EA. Carbohydrate ingestion does not alter carbohydrate or fat metabolism in human skeletal muscle during exercise. J Physiol. 2000;529(3):837-847. 
11. Graham TE, Spriet LL. Performance and metabolic response to a high caffeine dose during prolonged exercise. J Appl Physiol. 1995;71(6):2292-2298.

12. Graham TE, Spriet LL. Metabolic, catecholamine, and exercise performance responses to various doses of caffeine. J Appl Physiol. 1991;78(3):867-874.

13. Graham-Paulson TS, Perret C, Watson P, Goosey-Tolfrey VL. Improvement in sprint performance in wheelchair sportsmen with caffeine supplementation. Int J Sport Physiol Perform. 2015;11(2):214-220.

14. Greer F, Friars D, Graham TE. Comparison of caffeine and theophylline ingestion: exercise metabolism and endurance. J Appl Physiol. 2000;89:1837-1844.

15. Halstead LS. Feldman S, Claus-Walker J, Patel VC. Drug absorption in spinal cord injury. Arch Phys Med Rehab. 1985;66(5):298-301.

16. Holland DT, Godfredsen KA, Page T, Connor JD. Simple high-performance liquid chromatography method for the simultaneous determination of serum caffeine and paraxanthine following rapid sample preparation. J Chromatogr B. 1991;707:105-110.

17. Houtman S, Oeseburg B, Hopman MTE. Blood volume and haemoglobin after spinal cord injury. Am J Physical Med Rehab, 2000;79:260-265.

18. Kao C, Ho Y, Changlai S, Ding H. Gastric emptying in spinal cord injury patients. Digest Dis Sci. 1999;44:1512-1515.

19. Keisler BD, Armsey TD. Caffeine as an ergogenic aid. Curr Sports Med Rep. 2006;5:168-177.

20. Kirschblum SC, Burns, SP, Biering-Sorensen F. International standards for neurological classification of spinal cord injury (revised 2011). J Spinal Cord Med. 2011;34:535-546.

21. Landrum RE. College students' use of caffeine and its relationship to personality. Coll Stud J. 1992;26:151-155. 
22. Marsh GD, McFadden RG, Nicholson RL, Leasa DJ, Thompson TR. Theophylline delays skeletal muscle fatigue during progressive exercise. Am J Respir Crit Care Med. 1993;147(4):876-879.

23. Mestre H, Alkon T, Salazar S, Ibarra A. Spinal cord injury sequelae alter drug pharmacokinetics: an overview. Int Spinal Cord Soc. 2011;49:955-960.

24. Mohr T, Van Soeren M, Graham TE, Kjaer M. Caffeine ingestion and metabolic responses of tetraplegic humans during electrical cycling. J Appl Physiol. 1998;85:979-985.

25. Monroe MB, Tataranni PA, Pratley R, Manore MM, Skinner JS, Ravussin E. Lower daily energy expenditure as measured by a respiratory chamber in subjects with spinal cord injury compared with control subjects. Am J Clin Nutr, 1998;68:1223-1227.

26. Paton C, Costa V Guglielmo L. Effects of caffeine chewing gum on race performance and physiology in male and female cyclists. J Sport Sci. 2015;10:1076-1083.

27. Paulson TAW, Goosey-Tolfrey VL, Lenton JP, Leicht CA, Bishop NC. Spinal cord injury level and the circulating cytokine response to strenuous exercise. Med Sci Sports Exerc. 2013;45(9):1649-1655.

28. Raguso CA, Coggan AR, Sidossis LS, Gastaldelli A, Wolfe RR. Effect of theophylline on substrate metabolism during exercise. Metab. 1996;45(9):1153-1160.

29. Ryan WJ, Kim C-H, Muller MD. Low-dose caffeine administered in chewing gum does not enhance cycling to exhaustion. J Str Cond Res. 2012;26)3):844-850.

30. Sauerbeck AD, Laws L, Bandara VVR, Popovich PG, Haughey NJ, McTiguue DM. Spinal cord injury causes chronic liver pathology in rats. $J$ Neurotrauma. 2015;32:159-169. 
31. Schmid A, Huonker M, Stahl F. Free plasma catecholamines in spinal cord injured persons with different injury levels at rest and during exercise. $J$ Autonom Nerv Syst. 1998;68:96-100.

32. Segal JL, Brunnemann SR, Gordon SK, Eltorai IM. The absolute bioavailability of oral theophylline in patients with spinal cord injury. Pharmacotherapy. 1986; 6(1): 26-29.

33. Skinner TL, Jenkins DG, Coombes JS, Taafe, DR, Leveritt MD. Dose response of caffeine on 2000-m rowing performance. Med Sci Sports Exerc. 2010;42(3):571-576.

34. Skinner TL, Jenkins DG, Coombes JS, Taafe DR, Leveritt MD. Coinciding exercise with peak serum caffeine does not improve cycling performance. J Sci Med Sport. 2013;16:54-59.

34.35. Skinner TL, Jenkins, DG, Leveritt MD, McGorm A, Bolam KA, Coombes JS, Taaffe, DR. Factors influencing serum caffeine concentrations following caffeine ingestion. J Sci Med Sport. 2014; 17:516-520.

35.36. Spungen AM, Adkins RH, Stewart CA. Factors influencing body composition in persons with a spinal cord injury: a cross-sectional study. J Appl Physiol. 2003;95:2398-2407.

36.37. Statland BE, Demas TJ. Serum caffeine half-lives. Healthy subjects vs. patients having alcoholic hepatic disease. Am J Clin Pathol. 1980;73(3):390-393.

37.38. Van Soeren M, Mohr T, Kjaer M Graham TE. Acute effects of caffeine ingestion at rest in humans with impaired epinephrine responses. J Appl Physiol. 1996; 80: 999-1005.

38.39. Yi C-X, la Fleur SE, Fliers E, Kalsbeek A. The role of the autonomic nervous liver innervation in the control of energy metabolism. Biochimica et Biophysica Acta. 2010;1802:416-431. 


\section{Tables}

473 Table 1. Participants’ characteristics.

474

\section{$475 \quad$ Figures}

476 Figure 1. Schematic of the experimental protocol.

477 Figure 2. Mean \pm SD plasma caffeine concentration following the consumption of $3 \mathrm{mg} \cdot \mathrm{kg}^{-1}$ 478 caffeine anhydrous (Aa). Individual data from able-bodied participants (B $\underline{b})$, and participants 479 with paraplegia $\left(\epsilon_{\underline{c}}\right)$ and tetraplegia $\left(\mathrm{Bd}_{\mathrm{d}}\right)$ (dotted/bold lines represent individuals with an 480 ASIA A/B classification).

481 Figure 3. Plasma adrenaline (a), noradrenaline (b), free fatty acid (Aㄷ), lactate (Bd)), glucose 482 (Ge), noradrenaline (D) and adrenaline (E) concentrations (mean \pm SD) following the 483 consumption of $3 \mathrm{mg} \cdot \mathrm{kg}^{-1}$ caffeine anhydrous in able-bodied (AB) individuals and individuals 484 with paraplegia (PARA) and tetraplegia (TETRA). * significant main effect for group; † 485 significant time by group interaction effect.

486 - ${ }^{a}$ Significantly different to baseline values, ${ }^{b}$ significantly different to 60 min, ${ }^{\epsilon}$ significantly 487 different to 90 min. 